\title{
MATCHMAKING AND TESTING FOR EXPONENTIALITY IN THE M/G/ $\infty$ QUEUE
}

\author{
RUDOLF GRÜBEL *** AND \\ HENDRIK WEGENER, ${ }^{* * * *}$ Leibniz Universität Hannover
}

\begin{abstract}
Customers arrive sequentially at times $x_{1}<x_{2}<\cdots<x_{n}$ and stay for independent random times $Z_{1}, \ldots, Z_{n}>0$. The $Z$-variables all have the same distribution $Q$. We are interested in situations where the data are incomplete in the sense that only the order statistics associated with the departure times $x_{i}+Z_{i}$ are known, or that the only available information is the order in which the customers arrive and depart. In the former case we explore possibilities for the reconstruction of the correct matching of arrival and departure times. In the latter case we propose a test for exponentiality.
\end{abstract}

Keywords: Asymptotics; Kendall's $\tau$; log-concave density; log-convex density; queue; prediction; permutation

2010 Mathematics Subject Classification: Primary 60K25

Secondary 62M07; 62M 20

\section{Introduction}

In order to explain the problems discussed in the present paper we consider the years of birth and death of the composers J. S. Bach (1685-1750), Haydn (1732-1809), Mozart (17561791), Beethoven (1770-1827), Schubert (1797-1828), Brahms (1833-1897), and Tchaikovsky (1840-1893). The top diagram of Figure 1 shows the birth and death years on the upper and lower time axes respectively; the connecting lines indicate the correct matching, i.e. the true year of death associated with any particular year of birth. Suppose now that the correct matching is not known and that we only have the order statistics associated with the birth times and times of death, and that the lifetime distribution $Q$ is known. Can we reconstruct the matching? Or, suppose that only the order of arrivals and departures is known, and that $Q$ is not known. In our running example this would lead to $(1,2,1,3,4,3,5,2,4,5,6,7,7,6)$, where the first occurrence of a number signifies birth, the second death. Is there still some information about $Q$ in this severely reduced data set?

The first type of data loss typically appears whenever the number of 'customers' in a 'system' is recorded, where the meaning of these words depends on the respective application. For our running example, this is illustrated by the bottom diagram of Figure 1, where the number of composers alive at time $t$ is given as a function of $t$ : knowing the function is obviously equivalent to knowing the ordered arrival and departure data. If arrivals occur at the time instances of a Poisson process with constant rate then we obtain an initial path segment of a queue of the type $\mathrm{M} / \mathrm{G} / \infty$ in D. G. Kendall's notation (see, e.g. Chapter III 1b of [2]).

Received 3 June 2010; revision received 24 November 2010.

* Postal address: Institut für Mathematische Stochastik, Leibniz Universität Hannover, Postfach 6009, D-30060

Hannover, Germany.

** Email address: rgrubel@stochastik.uni-hannover.de

*** Email address: wegener@ stochastik.uni-hannover.de 

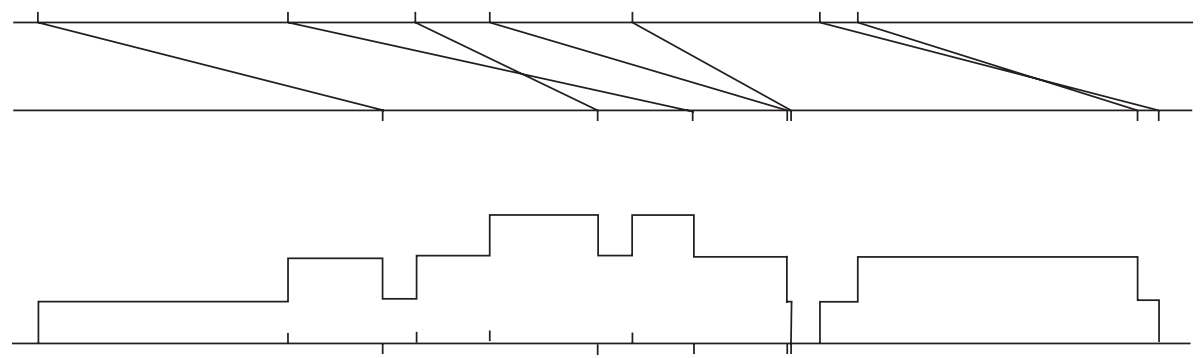

Figure 1: Composer data.

Formally, we have arrival times $x_{1}<x_{2}<\cdots<x_{n}$, which we first regard as fixed. At each of these instances a delay period (or lifetime or service time) begins; we assume that these are nonnegative, independent, and identically distributed random variables $Z_{1}, \ldots, Z_{n}$. We call the distribution $Q$ of the $Z$-variables the delay distribution and generally assume that it has a density $f$ with respect to the Lebesgue measure. Let $\tilde{Y}_{i}=x_{i}+Z_{i}, 1 \leq i \leq n$, be the departure times (or times of death, etc.), and let $Y_{1}, \ldots, Y_{n}$ be the increasing order statistics associated with $\tilde{Y}_{1}, \ldots, \tilde{Y}_{n}$. With probability 1 , there exists a unique random permutation $\Pi$ in the set $\mathbb{S}_{n}$ of all permutations of $\{1, \ldots, n\}$ such that $\tilde{Y}_{i}=Y_{\Pi_{i}}, 1 \leq i \leq n$. Furthermore, $V_{i}:=\#\left\{1 \leq j<i: \tilde{Y}_{j}>x_{i}\right\}$ denotes the number of customers that are in the system at the time of arrival of the $i$ th customer, $1 \leq i \leq n$.

In Section 2 we consider the problem of predicting or approximating the value of $\Pi$ on the basis of $x=\left(x_{1}, \ldots, x_{n}\right)$ and $Y=\left(Y_{1}, \ldots, Y_{n}\right)$, where we regard $Q$ as known. We deal with maximum a posteriori and minimum distance reconstructions. In Section 3 we introduce and discuss a test for the hypothesis that $Q$ is an exponential distribution, now on the basis of the data $(\pi, v)$, where $\pi=\left(\pi_{1}, \ldots, \pi_{n}\right)$ is the value of the matching $\Pi$, and $v=\left(v_{1}, \ldots, v_{n}\right)$ is the value of the random vector $V=\left(V_{1}, \ldots, V_{n}\right)$. In Section 4 we examine the asymptotic behaviour of our procedures in the case of Poisson process arrivals.

The situation considered in the present paper is similar to the broken sample problem introduced in [12], where a sample $\left(X_{1}, Y_{1}\right), \ldots,\left(X_{n}, Y_{n}\right)$ from a distribution on $\mathbb{R}^{k} \times \mathbb{R}^{l}$ is reduced to the respective multisets of the $X$-and $Y$-values; see also [4] and [11]. In [12] it is assumed that the distribution has a density $f$ that can be written as $f(x, y)=\alpha(x) \beta(y) \exp (\gamma(x) \delta(y))$ with suitable functions $\alpha, \beta, \gamma$, and $\delta$; in view of our general assumption $x<y$ this is not possible for delay data. In [4] and [11] related statistical questions are investigated, such as the possibility of consistent estimation of the correlation coefficient for bivariate normal distributions. In the delay situation, estimation of $Q$ on the basis of the ordered arrival and departure data has been considered in the M/G/ $\infty$ context in [8] and [14]; see also the references given therein. The problem that originally motivated the present investigation comes from insurance mathematics: in the context of multivariate risk theory the class of models introduced in [6] incorporates dependence between the components by assuming that original events may trigger later claims in different business lines. The associated ruin functions then depend on the delay times. Finally, from a very general point of view, the framework considered here is somewhat similar to the situation with 'decompounding' (see [9]), in the sense that an inverse problem arises that may be of interest in its own right.

In addition to the model variables defined above, the following notation will be used. We write $\mathcal{L}(X)$ for the distribution of a random variable $X$, occasionally abbreviating $\mathcal{L}(X)=Q$ to $X \sim Q$. We assume throughout the paper that the delay distribution $Q$ has a density $f$ 
that is strictly positive on the whole of $\mathbb{R}_{+}$. In our results we further assume that $f$ is logconvex or log-concave. In order to have compact notation, we write $\mathcal{P}_{\text {lcv }}$ and $\mathcal{P}_{\text {lcc }}$ for the class of distributions with log-convex and log-concave densities, respectively, and $\mathcal{P}_{\mathrm{lcv}}^{\circ}$ and $\mathcal{P}_{\text {lcc }}^{\circ}$ if these are strictly log-convex or strictly log-concave. These families are often used in the economics literature (see [1] and [3]), but also appear in reliability theory (see [5, p. 75ff.]). Two prominent examples, especially in connection with lifetimes and service times, are the Weibull and the gamma distributions, with densities

$$
f_{W}(z)=f_{W}(z \mid \beta, \eta)=\beta \eta^{\beta} z^{\beta-1} \exp \left(-(\eta z)^{\beta}\right), \quad z>0
$$

and

$$
f_{\Gamma}(z)=f_{\Gamma}(z \mid \beta, \eta)=\frac{1}{\Gamma(\beta)} \eta^{\beta} z^{\beta-1} \exp (-\eta z), \quad z>0,
$$

respectively. It is known (and easy to check) that these are strictly log-concave if $\beta>1$ and strictly $\log$-convex if $\beta<1$. With $\beta=1$ we obtain the exponential distribution $\operatorname{Exp}(\eta)$ in the Weibull as well as in the gamma case; indeed, in the above notation,

$$
\mathcal{P}_{\text {lcv }} \cap \mathcal{P}_{\text {lcc }}=\{\operatorname{Exp}(\eta): \eta>0\} .
$$

For further examples of log-convex and log-concave distributions, and of distributions that are neither, we refer the reader to [3].

\section{Matchmaking}

In this section we regard $x$ as fixed (and known), our data consist of a realization $y$ of $Y$, and we are interested in the (unknown) value $\pi$ of the matching $\Pi$. As the $Z$-variables are assumed to be strictly positive, $\pi$ must be an element of the set

$$
\mathbb{S}_{n}^{\circ}=\mathbb{S}_{n}^{\circ}(x, y):=\left\{\pi \in \mathbb{S}_{n}: y_{\pi_{i}}>x_{i} \text { for } i=1, \ldots, n\right\}
$$

of permissible permutations. We use $\pi=\left(\pi_{1}, \ldots, \pi_{n}\right)$ as a notation for the permutation $i \mapsto \pi_{i}$, and we recall that the elements of $\mathbb{S}_{n}$ can be coded by the relative ranks of these values,

$$
\Phi: \mathbb{S}_{n} \rightarrow{ }_{i=1}^{n}\{1, \ldots, i\}, \quad \Phi_{i}(\pi)=\#\left\{1 \leq j \leq i: \pi_{j} \leq \pi_{i}\right\} .
$$

In view of the support assumption on $Q$ all elements of the set $\mathbb{S}_{n}^{\circ}$ appear with positive probability. It is easy to see that

$$
V_{i}=i-1-\#\left\{1 \leq j \leq n: Y_{j}<x_{i}\right\},
$$

which means that $V$ is a function of $x$ and $Y$; in particular, we know its value $v=\left(v_{1}, \ldots, v_{n}\right)$. At the time of the $i$ th arrival $i-1-v_{i}$ departures have already taken place, so the range of values for the relative rank of the $i$ th departure time within the departures associated with the earlier arrivals is the set $\left\{i-v_{i}, \ldots, i\right\}$, and we can define a bijective mapping

$$
\Psi: W_{n}:=\chi_{i=1}^{n}\left\{i-v_{i}, \ldots, i\right\} \rightarrow \mathbb{S}_{n}^{\circ}
$$

by the requirement that $\Psi^{-1}=\Phi$ on $\mathbb{S}_{n}^{\circ}$ with $\Phi$ as in (2.1). Obviously,

$$
\Psi(1,2, \ldots, n)=\mathrm{id}:=(1,2, \ldots, n) \in \mathbb{S}_{n}^{\circ},
$$


which is the permissible permutation where customers depart in the order of their arrival. Also, as a consequence of the existence of a bijective mapping, the number of permissible permutations is a simple function of the vector $v=\left(v_{1}, \ldots, v_{n}\right)$ :

$$
\# \mathbb{S}_{n}^{\circ}=\prod_{i=1}^{n}\left(1+v_{i}\right)
$$

The joint distribution of $Y$ and $\Pi$ has density

$$
g\left(y_{1}, \ldots, y_{n}, \pi_{1}, \ldots, \pi_{n}\right)=\prod_{i=1}^{n} f\left(y_{\pi_{i}}-x_{i}\right)
$$

for $y_{1}<y_{2}<\cdots<y_{n}$ and $\pi \in \mathbb{S}_{n}^{\circ}$, where the density is with respect to the product of the $n$-dimensional Lebesgue measure on $\mathbb{R}^{n}$ and the counting measure on $\mathbb{S}_{n}$. Regarding the conditional density of $\Pi$ given $Y=y$ as a function of $\pi$ we call $\hat{\pi} \in \mathbb{S}_{n}$ a maximum a posteriori (MAP) reconstruction or prediction (or perhaps retrodiction) of the value $\pi$ of $\Pi$ if it maximizes this function, or, equivalently,

$$
\pi \mapsto \sum_{i=1}^{n} \log f\left(y_{\pi_{i}}-x_{i}\right)
$$

on $\mathbb{S}_{n}^{\circ}$. By design, $\hat{\Pi}:=\hat{\pi}(Y)$ maximizes the probability that the unknown matching $\Pi$ is equal to $\hat{\Pi}$ among all functions of the data $Y$. Our first result gives these maximizers for the classes of distributions introduced at the end of Section 1.

Theorem 2.1. (a) If $Q=\operatorname{Exp}(\eta)$ then all elements of $\mathbb{S}_{n}^{\circ}$ are $M A P$ predictors.

(b) If $Q \in \mathcal{P}_{\mathrm{lcc}}$ then $\hat{\pi}=\mathrm{id}$ is a MAP predictor; if $Q \in \mathcal{P}_{\mathrm{lcc}}^{\circ}$ then the MAP predictor is unique.

(c) If $Q \in \mathcal{P}_{\mathrm{lcv}}$ then a MAP predictor is given recursively by

$$
\begin{aligned}
\hat{\pi}_{n} & =\min \left\{j: y_{j}>x_{n}\right\}, \\
\hat{\pi}_{i} & =\min \left\{j: y_{j}>x_{i}, j \notin\left\{\hat{\pi}_{k}: k>i\right\}\right\}, \quad 1 \leq i<n .
\end{aligned}
$$

Moreover, if $Q \in \mathcal{P}_{\mathrm{lcv}}^{\circ}$ then the MAP predictor is unique.

Proof. For exponential distributions, we obtain

$$
\sum_{i=1}^{n} \log f\left(y_{\pi_{i}}-x_{i}\right)=n \log \eta-\eta \sum_{i=1}^{n}\left(y_{\pi_{i}}-x_{i}\right)=n \log \eta-\eta\left(\sum_{i=1}^{n} y_{i}-\sum_{i=1}^{n} x_{i}\right),
$$

which does not depend on $\pi$.

For the proofs of the remaining parts, we need some properties of $\mathbb{S}_{n}^{\circ}$. The set $W_{n}$ defined in (2.3) carries a canonical digraph structure, with a pair $(w, \tilde{w})$ of elements of $W_{n}$ being a directed edge if and only if there is a $j \in\{1, \ldots, n\}$ such that

$$
\tilde{w}_{i}= \begin{cases}w_{j}+1 & \text { if } i=j \\ w_{i} & \text { otherwise. }\end{cases}
$$


There are no loops, the vector $\bar{w}=(1,2, \ldots, n)$ is at the top, and $\underline{w}=\left(1,2-v_{2}, \ldots, n-v_{n}\right)$ is at the bottom. The digraph is connected in the sense that, for each $w \in W_{n}$, there is a path connecting the bottom to $w$ and a path connecting $w$ to the top. The mapping $\Psi$ transports this structure to $\mathbb{S}_{n}^{\circ}$ if we take $(\pi, \tilde{\pi})$ to be an edge in $\mathbb{S}_{n}^{\circ}$ if and only if $(w, \tilde{w})$ is an edge in $W_{n}$, with $\pi=\Psi(w)$ and $\tilde{\pi}=\Psi(\tilde{w})$. It is easy to check that the transition from $w$ to $\tilde{w}$ in (2.5) corresponds to the removal of an inversion in $\pi=\Psi(w)$ : position $j$ is swapped with position $i<j$, where customer $i$ is the first to leave after customer $j$ (we have $w_{j}<j$, so this set is not empty).

The properties that we need can now be formulated as follows. First, if $(\pi, \tilde{\pi})$ is a directed edge then $\tilde{\pi}$ arises from $\pi$ by a transposition that removes an inversion; second, $\mathbb{S}_{n}^{\circ}$ is connected in the sense that any permissible permutation $\pi$ can be transformed into $\Psi(\underline{w})$ or $\Psi(\bar{w})$ by a finite number of transpositions without leaving $\mathbb{S}_{n}^{\circ}$, i.e. with all intermediate results being permissible. The algorithm in part (c) is easily seen to provide the bottom $\Psi(\underline{w})$ of $\mathbb{S}_{n}^{\circ}$.

Now suppose that $f$ is $\log$-concave and that $(\pi, \tilde{\pi})$ is an edge in $\mathbb{S}_{n}^{\circ}$, so that $\tilde{\pi}=\pi \circ \tau(i, j) \in$ $\mathbb{S}_{n}^{\circ}$ for some transposition $\tau(i, j)$. We may assume that $i<j$, and then $\pi_{i}>\pi_{j}$. From the fact that $\pi, \tilde{\pi} \in \mathbb{S}_{n}^{\circ}$ we obtain $x_{i}<x_{j}<y_{\pi_{j}}<y_{\pi_{i}}$; in particular, $a:=x_{j}-x_{i}, b:=y_{\pi_{j}}-x_{j}$, and $c:=y_{\pi_{i}}-y_{\pi_{j}}$ are all positive. We need to maximize the function $h: \mathbb{S}_{n}^{\circ} \rightarrow \mathbb{R}$,

$$
h(\pi):=\sum_{k=1}^{n} \log f\left(y_{\pi_{k}}-x_{k}\right),
$$

and we obtain

$$
\begin{aligned}
h(\pi)-h(\tilde{\pi})= & \log \left(f\left(y_{\pi_{i}}-x_{i}\right)\right)+\log \left(f\left(y_{\pi_{j}}-x_{j}\right)\right)-\log \left(f\left(y_{\pi_{j}}-x_{i}\right)\right) \\
& -\log \left(f\left(y_{\pi_{i}}-x_{j}\right)\right) \\
= & \log (f(a+b+c))+\log (f(b))-\log (f(a+b)) \\
& -\log (f(b+c)) .
\end{aligned}
$$

For $\log f$ concave, this is easily seen to be less than or equal to 0 ; in the case of strict concavity it is even strictly negative (see also Lemma 1 of [1]). This means that by removing an inversion we (strictly) increase the value of $h$; in view of the structure of $\mathbb{S}_{n}^{\circ}$ this implies both statements in (b).

For $\log f$ (strictly) convex, the difference is (strictly) positive; hence, we obtain the (unique) MAP in situation (c) by moving to the bottom, which again is possible without leaving $\mathbb{S}_{n}^{\circ}$.

Remark 2.1. (a) The algorithm in Theorem 2.1(c) assigns to each arrival the earliest possible departure, working backwards from the latest arrival, whereas, with the solution $\hat{\pi}=$ id in part (b), departures are delayed as long as possible. Note that the MAP does not depend on the data in the log-concave case; for log-convex densities, it depends on the data $y$ only via the set $\mathbb{S}_{n}^{\circ}$ of permissible distributions.

(b) For other popular distributions that are neither log-convex nor log-concave, such as the lognormal distribution, we do not have explicit expressions for the MAP predictor(s) $\hat{\pi}$. Algorithmically, the maximization of the function in (2.4) can be seen as an instance of the assignment problem (see, e.g. [15, Chapter 13]), which can be solved with complexity $O\left(n^{3}\right)$; hence, we can still find the solutions in a reasonable amount of time if $n$ is not too large. This is an improvement over the naive approach of working through the whole of $\mathbb{S}_{n}^{\circ}$ element by element, as this set may be quite large even for moderate values of $n$. 
It should be clear that a reconstruction in the sense of $\mathrm{P}(\hat{\Pi}=\Pi)$ being large is somewhat unrealistic in all but extreme cases (such as, in queueing terminology, cases with a very low traffic intensity). Instead, we could aim for a prediction that is close to the true value in the mean. This requires some loss function, which in turn could be based on a notion of distance. Formally, we have a metric $d$ on $\mathbb{S}_{n}$ and we call a function $\phi$ of the data $y$ a minimum mean distance (MMD) predictor with respect to $d$ if $\phi$ minimizes $\mathrm{E} d(\Pi, \phi(Y))$ among all measurable functions $\phi: \mathbb{R}^{n} \rightarrow \mathbb{S}_{n}$. If the transition kernel $K(y, \cdot)$ is a version of $\mathcal{L}(\Pi \mid Y=y)$ then we obtain an MMD predictor by minimizing $\pi \mapsto \int d(\pi, \sigma) K(y, \mathrm{~d} \sigma)$ for the individual $y$-values. Of course, MAP prediction for discrete random variables can also be seen as minimizing an expected distance, if we let the distance between $\pi$ and $\sigma$ be 1 if $\pi \neq \sigma$, and 0 otherwise (the discrete metric). There are obvious analogies with concepts from frequentist and Bayesian statistics; indeed, what we call MAP prediction occasionally appears as 'maximum likelihood prediction' in the literature.

Our basic reference for distances of permutations and their statistical relevance is [13, Chapter 6]. We now consider minimum mean distance prediction of $\Pi$ if we measure distance by M. G. Kendall's $\tau$. The standard definition of $d_{\tau}(\pi, \sigma)$ is as the minimum number of transpositions $\tau(i, i+1), 1 \leq i<n$, of direct neighbours required to turn $\pi^{-1}$ into $\sigma^{-1}$. There are various other, equivalent, definitions; for us, it will be convenient to use

$$
d_{\tau}(\sigma, \pi)=\sum_{i=1}^{n-1} \sum_{j=i+1}^{n} D_{i, j}
$$

with

$$
D_{i, j}=D_{i, j}(\sigma, \pi):= \begin{cases}1 & \text { if }\left(\sigma_{i}-\sigma_{j}\right)\left(\pi_{i}-\pi_{j}\right)<0, \\ 0 & \text { otherwise. }\end{cases}
$$

Here $D_{i, j}(\sigma, \pi)$ indicates whether or not $\pi$ and $\sigma$ assign different orders to the values $i$ and $j$. In particular, with the bijection $\Psi$ in (2.1) and (2.3), we have

$$
d_{\tau}(\mathrm{id}, \pi)=\sum_{i=1}^{n}\left(i-w_{i}\right) \quad \text { if } \pi=\Psi\left(w_{1}, \ldots, w_{n}\right) .
$$

Note that the Cayley distance arises if instead of neighbour transpositions we allow all transpositions $\tau(i, j), 1 \leq i<j \leq n$. Apart from being more detailed in a sense, Kendall's $\tau$ also seems to us to be more appropriate in the present context as the delay problem makes use of the ordering of the set on which the permutations work via the ordering in time of the events in question.

Stated somewhat informally, the proof of Theorem 2.1 shows that, for log-concave densities, undoing an inversion increases the a posteriori probability, and that we obtain a decrease in the log-convex case. For the first of these, it turns out that the MAP predictor is also the MMD predictor; for the corresponding statement in the log-convex case, we need an additional assumption. Note that, with $\mathbb{S}_{n}^{\circ}=\mathbb{S}_{n}$, the permutation in Theorem 2.1(b) is the same as the permutation defined in Theorem 2.1(c).

Theorem 2.2. (a) If $Q \in \mathcal{P}_{\text {lcc }}$ then $\phi(Y) \equiv \mathrm{id}$ is an $M M D$ predictor for $\Pi$ with respect to Kendall's $\tau$-distance. If $Q \in \mathcal{P}_{\mathrm{lcc}}^{\circ}$ then the MMD predictor is unique.

(b) Suppose that $\mathbb{S}_{n}^{\circ}=\mathbb{S}_{n}$. If $Q \in \mathcal{P}_{\mathrm{lcv}}$ then $\phi(Y) \equiv \hat{\pi}$ with $\hat{\pi}_{i}=n-i+1$ for $i=1, \ldots, n$ is an MMD predictor for $\Pi$ with respect to Kendall's $\tau$-distance. If $Q \in \mathcal{P}_{\mathrm{lcc}}^{\circ}$ then the $M M D$ predictor is unique. 
Proof. (a) Let $K(y, \cdot)$ be the conditional distribution of $\Pi$ given $Y=y$. The computation in (2.6) shows that, for log-concave densities, and with

$$
B_{i, j}:=\left\{\pi \in \mathbb{S}_{n}: \pi_{i}>\pi_{j}\right\}, \quad 1 \leq i<j \leq n,
$$

we have

$$
\left\{\pi \circ \tau(i, j): \pi \in B_{i, j} \cap \mathbb{S}_{n}^{\circ}\right\} \subset B_{i, j}^{\mathrm{c}} \cap \mathbb{S}_{n}^{\circ}
$$

as well as

$$
K(y,\{\pi \circ \tau(i, j)\}) \geq K(y,\{\pi\}) \quad \text { for all } \pi \in B_{i, j} \cap S_{n}^{\circ} .
$$

In particular,

$$
K\left(y,\left\{\pi: \pi_{i}>\pi_{j}\right\}\right) \leq K\left(y,\left\{\pi: \pi_{i}<\pi_{j}\right\}\right) \text { for all } i, j \text { with } 1 \leq i<j \leq n,
$$

as $\pi \mapsto \pi \circ \tau(i, j)$ maps $B_{i, j} \cap \mathbb{S}_{n}^{\circ}$ one-to-one onto a subset of $\left\{\pi \in \mathbb{S}_{n}^{\circ}: \pi_{i}<\pi_{j}\right\}$.

We now use the representation given by (2.7) and (2.8). Clearly, for any $\sigma \in \mathbb{S}_{n}^{\circ}$, and again with $1 \leq i<j \leq n$,

$$
\mathrm{E}\left[D_{i, j}(\sigma, \Pi) \mid Y\right]= \begin{cases}K\left(Y,\left\{\Pi_{i}>\Pi_{j}\right\}\right) & \text { if } \sigma_{i}<\sigma_{j} \\ K\left(Y,\left\{\Pi_{i}<\Pi_{j}\right\}\right) & \text { if } \sigma_{i}>\sigma_{j}\end{cases}
$$

which reduces to $K\left(Y,\left\{\Pi_{i}>\Pi_{j}\right\}\right)$ if $\sigma=\mathrm{id}$. With (2.12), this leads to

$$
\mathrm{E} D_{i, j}(\sigma, \Pi)=\mathrm{E}\left[\mathrm{E}\left[D_{i, j}(\sigma, \Pi) \mid Y\right]\right] \geq \mathrm{E}\left[\mathrm{E}\left[D_{i, j}(\mathrm{id}, \Pi) \mid Y\right]\right]=\mathrm{E} D_{i, j}(\mathrm{id}, \Pi)
$$

so that

$$
\mathrm{E} d_{\tau}(\sigma, \Pi)=\sum_{i=1}^{n-1} \sum_{j=i+1}^{n} \mathrm{E} D_{i, j}(\sigma, \Pi) \geq \sum_{i=1}^{n-1} \sum_{j=i+1}^{n} \mathrm{E} D_{i, j}(\mathrm{id}, \Pi)=\mathrm{E} d_{\tau}(\mathrm{id}, \Pi)
$$

Again, for strictly log-concave densities, the inequality will be strict.

(b) In the log-convex case, the inequality in (2.11) is reversed, and the additional assumption that $\mathbb{S}_{n}^{\circ}=\mathbb{S}_{n}$ implies that we have equality in (2.10). Replacing $\sigma=$ id by $\sigma=\hat{\pi}$ we can now proceed as in the proof of the first part of the theorem.

Remark 2.2. (a) It follows from the first part of the proof of Theorem 2.1 that, for exponential delay distributions, the conditional distribution of $\Pi$ given $Y$ is the discrete uniform distribution on the set of permissible permutations; we abbreviate this to

$$
\mathcal{L}(\Pi \mid Y)=\operatorname{unif}\left(\mathbb{S}_{n}^{\circ}\right) .
$$

Indeed, as we regard arrival times as fixed, $\mathbb{S}_{n}^{\circ}$ is a function of $Y$. Moreover, the distribution of the distance between the predictor that is best in the sense of Theorem 2.2(a) and the true matching can then be written as a convolution of uniform distributions, i.e.

$$
\mathcal{L}\left(d_{\tau}(\mathrm{id}, \Pi) \mid Y\right)=\stackrel{\star}{\star=1}_{i=1}^{n} \operatorname{unif}\left(\left\{0, \ldots, V_{i}\right\}\right),
$$

where $V_{i}$ again denotes the number of customers that are in the system at the time of the $i$ th arrival. To see this, we use (2.9) and (2.13), and we note that uniformity on $\mathbb{S}_{n}^{\circ}$ implies uniformity on the domain of the bijective function $\Psi$ in (2.3). In particular,

$$
\mathrm{E}\left[d_{\tau}(\mathrm{id}, \Pi) \mid Y\right]=\frac{1}{2} \sum_{i=1}^{n} V_{i}, \quad \operatorname{var}\left[d_{\tau}(\mathrm{id}, \Pi) \mid Y\right]=\frac{1}{12} \sum_{i=1}^{n} V_{i}\left(V_{i}+2\right) .
$$

This will be taken up in Section 4. 
(b) In the exponential case we cannot expect uniqueness if $\mathbb{S}_{n}^{\circ}=\mathbb{S}_{n}$ as the distance is right invariant in the sense that $d_{\tau}(\pi, \sigma)=d_{\tau}(\pi \circ v, \sigma \circ \nu)$ for all $\pi, \sigma, v \in \mathbb{S}_{n}$. Even if $\mathbb{S}_{n}^{\circ} \neq \mathbb{S}_{n}$ the minimizer may not be unique. For example, in the case in which $n=3$ and $x_{1}<x_{2}<$ $y_{1}<x_{3}<y_{2}<y_{3}$ we have $\mathbb{S}_{3}^{\circ}=\{\mathrm{id},(1,3,2),(2,1,3),(3,1,2)\}$, and some straightforward computations show that, for $\sigma=\mathrm{id}$ and $\sigma=(2,1,3)$, the distance $d_{\tau}(\sigma, \Pi)$ to $\Pi \sim \operatorname{unif}\left(\mathbb{S}_{3}^{\circ}\right)$ has a binomial distribution with parameters 2 and $\frac{1}{2}$; for the other two permutations, the distance is uniformly distributed on $\{0,1,2,3\}$. Hence, there are two permutations that minimize $\sigma \mapsto$ $\mathrm{E}\left[d_{\tau}(\sigma, \Pi) \mid \mathbb{S}_{3}^{\circ}\right]$. Also, for exponential delay distributions, choosing a permutation at random will provide an optimal reconstruction from the MAP point of view. With MMD, this is no longer the case. With $\mathbb{S}_{3}^{\circ}$ as above, and $X$ and $\Pi$ independent and uniformly distributed on $\mathbb{S}_{3}^{\circ}$, we obtain the expected distances $\mathrm{E} d_{\tau}(\mathrm{id}, \Pi)=1$ and $\mathrm{E} d_{\tau}(X, \Pi)=\frac{5}{4}$.

(c) For the data $x=(1,2,4)$ and $y=(3,5,6)$, and with $Q$ the gamma distribution with parameters $\beta=\frac{1}{2}$ and $\eta=1$, we obtain the MMD predictor $\pi=(2,1,3)$, whereas the permutation $\hat{\pi}$ defined in Theorem $2.1(\mathrm{c})$ is given by $\hat{\pi}=(3,1,2)$. Hence, in contrast to the MAP situation, this $\hat{\pi}$ will not in general be the MMD predictor in the log-convex case-an additional assumption on the structure of $\mathbb{S}_{n}^{\circ}$ such as in Theorem 2.2(b) is needed.

(d) In analogy to the MAP case the question arises of how an MMD predictor could be obtained if the lifetime distribution is not of the type considered in Theorem 2.2. We define a matrix $A$ and a vector $b$, both indexed by the permissible permutations, by

$$
A=(a(\pi, \sigma))_{\pi, \sigma \in \mathbb{S}_{n}^{\circ}} \text { with } \quad a(\pi, \sigma):=d_{\tau}(\pi, \sigma) \text { for all } \pi, \sigma \in \mathbb{S}_{n}^{\circ},
$$

and

$$
b=\left(b_{\pi}\right)_{\pi \in \mathbb{S}_{n}^{\circ}} \quad \text { with } \quad b_{\pi}:=\prod_{i=1}^{n} f_{Z}\left(y_{\pi_{i}}-x_{i}\right) \text { for all } \pi \in \mathbb{S}_{n}^{\circ},
$$

respectively. Finding the MMD for a particular $y$ then amounts to finding a component of the vector $A b$ that has minimal value. We are not aware of a general algorithmic simplification in the style of Remark 2.1(b), but the distance minimization can be carried out separately for the busy periods; see also the proof of Theorem 4.2 below.

\section{A test of exponentiality}

We now turn to the situation where the original data are reduced to the order of arrivals and departures. Equivalently, we observe the value $\pi$ of the true matching $\Pi$ and the value $v$ of the random vector $V=\left(V_{1}, \ldots, V_{n}\right)$, where, again, $V_{i}$ denotes the number of customers that are in the system at the time of arrival of the $i$ th customer. If the delay times have a log-concave density then we know from the proof of Theorem 2.1 that the conditional density of $\Pi$ given $Y$ decreases if the number of inversions increases; it increases in the log-convex case. This motivates the use of $d_{\tau}$ (id, $\pi$ ) as a test statistic for testing exponentiality against log-convex or log-concave alternatives. In the queueing context such procedures test for $\mathrm{M} / \mathrm{M} / \infty$ within $\mathrm{M} / \mathrm{G} / \infty$.

For a formal definition, let $\alpha \in(0,1)$ be given and let $\phi_{+}: \mathbb{S}_{n} \times \mathbb{N}_{0}^{n} \rightarrow[0,1]$ be defined by

$$
\phi_{+}(\pi, v)= \begin{cases}1 & \text { if } d_{\tau}(\mathrm{id}, \pi)>c_{+}(\alpha), \\ \gamma_{+}(\alpha) & \text { if } d_{\tau}(\mathrm{id}, \pi)=c_{+}(\alpha), \\ 0 & \text { if } d_{\tau}(\mathrm{id}, \pi)<c_{+}(\alpha),\end{cases}
$$

where $c_{+}(\alpha)$ is the upper $\alpha$-quantile associated with $Q_{0}:=\mathcal{L}\left(d_{\tau}(\mathrm{id}, \Pi)\right), \Pi \sim \operatorname{unif}\left(\mathbb{S}_{n}^{\circ}\right)$, and 
$\gamma_{+}(\alpha) \in[0,1)$ is such that

$$
Q_{0}\left(\left(c_{+}(\alpha), \infty\right)\right)+\gamma_{+}(\alpha) Q_{0}\left(\left\{c_{+}(\alpha)\right\}\right)=\alpha .
$$

Similarly, we define another test function $\phi_{-}$by

$$
\phi_{-}(\pi, v)= \begin{cases}1 & \text { if } d_{\tau}(\mathrm{id}, \pi)<c_{-}(\alpha), \\ \gamma_{-}(\alpha) & \text { if } d_{\tau}(\mathrm{id}, \pi)=c_{-}(\alpha), \\ 0 & \text { if } d_{\tau}(\mathrm{id}, \pi)>c_{-}(\alpha)\end{cases}
$$

where $c_{-}(\alpha)$ is now the lower $\alpha$-quantile of $Q_{0}$ and $\gamma_{-}(\alpha)$ is such that

$$
Q_{0}\left(\left[0, c_{-}(\alpha)\right)\right)+\gamma_{-}(\alpha) Q_{0}\left(\left\{c_{-}(\alpha)\right\}\right)=\alpha .
$$

In both cases, the variable $v$ enters the test via $\mathbb{S}_{n}^{\circ}$; see (2.3). We point out that the critical values do not depend on $\eta$, which is a consequence of the fact that, for exponential delay distributions, the conditional distribution of $\Pi$, and, hence, of $d_{\tau}(\mathrm{id}, \Pi)$, does not depend on this parameter; see (2.13). In fact, this distribution is a convolution of discrete uniform distributions, which makes it easy to obtain the critical values. For small $n$, this can be done via Fourier transforms, together with the fast Fourier transform (FFT) algorithm. For moderate or large $n$ and $v_{i} \mathrm{~s}$, the normal approximation gives satisfying results; see also Section 4.

Our next theorem collects some properties of these tests. Statements (3.4) and (3.6) below imply that both are unbiased and that they are similar on the 'boundary' $\mathcal{P}_{\text {lcv }} \cap \mathcal{P}_{\text {lcc }}=\{\operatorname{Exp}(\eta)$ : $\eta>0\}$; see [16, Chapter 4.3]. Statements (3.5) and (3.7) below show that the tests are strongly unbiased 'inside' their respective hypothesis and alternative.

The random variables that appear in our model are all defined on some measurable space $(\Omega, \mathcal{A})$. We write $\mathrm{P}_{Q}$ for the probability measure on $(\Omega, \mathcal{A})$ under which the lifetimes are independent and have distribution $Q$, and we write $\mathrm{E}_{Q}$ to denote the expectation with respect to $\mathrm{P}_{Q}$.

Theorem 3.1. (a) With $\phi_{+}$as in (3.1),

$$
\mathrm{E}_{Q}\left[\phi_{+}(\Pi, V) \mid V\right] \begin{cases}=\alpha & \text { if } Q=\operatorname{Exp}(\eta) \text { for some } \eta>0 \\ \leq \alpha & \text { if } Q \in \mathcal{P}_{\mathrm{lcc}} \\ \geq \alpha & \text { if } Q \in \mathcal{P}_{\mathrm{lcv}}\end{cases}
$$

and

$$
\mathrm{E}_{Q} \phi_{+}(\Pi, V) \begin{cases}<\alpha & \text { if } Q \in \mathcal{P}_{\mathrm{lcc}}^{\circ}, \\ >\alpha & \text { if } Q \in \mathcal{P}_{\mathrm{lcv}}^{\circ}\end{cases}
$$

(b) Similarly, with $\phi_{-}$as in (3.3),

$$
\mathrm{E}_{Q}\left[\phi_{-}(\Pi, V) \mid V\right] \begin{cases}=\alpha & \text { if } Q=\operatorname{Exp}(\eta) \text { for some } \eta>0 \\ \leq \alpha & \text { if } Q \in \mathcal{P}_{\mathrm{lcv}}, \\ \geq \alpha & \text { if } Q \in \mathcal{P}_{\mathrm{lcc}}\end{cases}
$$

and

$$
\mathrm{E}_{Q} \phi_{-}(\Pi, V) \begin{cases}<\alpha & \text { if } Q \in \mathcal{P}_{\mathrm{lcv}}^{\circ} \\ >\alpha & \text { if } Q \in \mathcal{P}_{\mathrm{lcc}}^{\circ}\end{cases}
$$




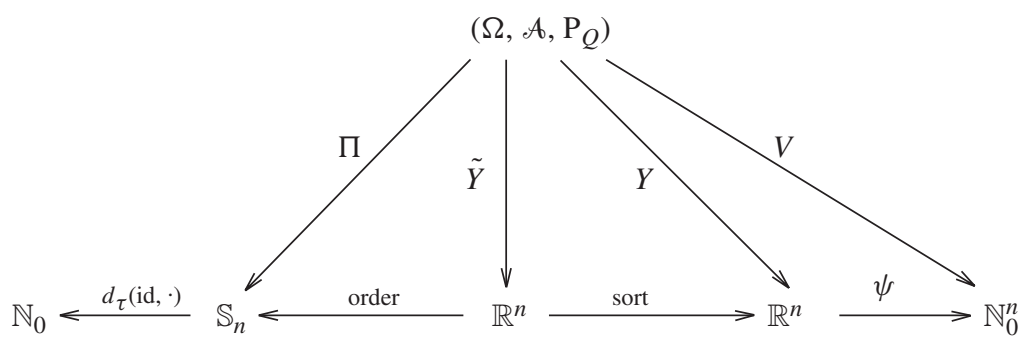

FIGURE 2: General structure of the model variables.

Proof. Let $\alpha \in(0,1)$ be given. The various random quantities are summarized in Figure 2, where 'order' and 'sort' are canonically defined (see, e.g. their use in the statistical language $\mathrm{R})$, and $\psi=\left(\psi_{1}, \ldots, \psi_{n}\right)$ is given by

$$
\psi_{i}\left(y_{1}, \ldots, y_{n}\right):=i-1-\#\left\{1 \leq j<n: y_{j}<x_{i}\right\}
$$

see also (2.2).

We abbreviate $\mathrm{P}_{Q}, \mathrm{E}_{Q}$ to $\mathrm{P}_{\eta}, \mathrm{E}_{\eta}$ if $Q=\operatorname{Exp}(\eta)$. Remark 2.2(a) and (3.2) lead to

$$
\mathrm{E}_{\eta}\left[\phi_{ \pm}(\Pi, V) \mid V\right]=\alpha \text { for all } \eta>0 .
$$

Integrating out the variable $V$ we see that the tests have the exact rejection probability $\alpha$ on $\{\operatorname{Exp}(\eta): \eta>0\}$

For the proof of the other assertions, we first consider the conditional distribution of $d_{\tau}(\mathrm{id}, \Pi)$ given $Y$. The structural considerations at the beginning of Section 2 imply that this distribution has support $\left\{0,1, \ldots, k_{\max }\right\}$ with

$$
k_{\max }=k_{\max }(Y):=\sum_{i=1}^{n} \psi_{i}(Y)=\sum_{i=1}^{n} V_{i} .
$$

Furthermore, the argument in the proof of Theorem 2.1 can be used to show that

$$
k \mapsto \frac{\mathrm{P}_{Q}\left(d_{\tau}(\mathrm{id}, \Pi)=k \mid Y\right)}{\mathrm{P}_{\eta}\left(d_{\tau}(\mathrm{id}, \Pi)=k \mid Y\right)}
$$

is strictly decreasing on $\left\{0,1, \ldots, k_{\max }\right\}$ if $Q \in \mathcal{P}_{\text {lcc }}^{\circ}$, provided that $Y$ is such that $k_{\max }(Y)>0$. An elementary argument (based, for example, on the proof of a comparison result for the likelihood ratio and stochastic order given in [17, p. 12]) shows that this implies that, again for $Q \in \mathcal{P}_{\mathrm{lcc}}^{\circ}$,

$$
\mathrm{P}_{Q}\left(d_{\tau}(\mathrm{id}, \Pi) \geq k \mid Y\right)<\mathrm{P}_{\eta}\left(d_{\tau}(\mathrm{id}, \Pi) \geq k \mid Y\right)
$$

for $k=1, \ldots, k_{\max }$. Furthermore, in (3.8) we may condition on $V$ instead of $Y$ in view of the fact that $V$ is a function of $Y$ (integrating over $\{y: \psi(y)=v\}$ leads to the conditional probability given $V=v$ ).

The strict stochastic ordering of the distributions of the test statistics in turn leads to a corresponding strict lexicographic ordering of the pairs $\left(c_{ \pm}(\alpha), \gamma_{ \pm}(\alpha)\right)$ derived from the distribution of $d_{\tau}$ (id, $\Pi$ ) under $\mathrm{P}_{Q}$ and $\mathrm{P}_{\eta}$; hence,

$$
\begin{array}{ll}
\mathrm{E}_{Q}\left[\phi_{+}(\Pi, V) \mid V=v\right]<\alpha & \text { for all } Q \in \mathcal{P}_{\mathrm{lcc}}^{\circ}, v \neq 0, \\
\mathrm{E}_{Q}\left[\phi_{-}(\Pi, V) \mid V=v\right]>\alpha & \text { for all } Q \in \mathcal{P}_{\mathrm{lcc}}^{\circ}, v \neq 0 .
\end{array}
$$


Here we have written 0 for the $n$-vector with all components equal to 0 . Note that $v \neq 0$ is equivalent to $\# \mathbb{S}_{n}^{\circ}>1$. As the $\tilde{Y}$-variables can take arbitrarily large values, we always have $\mathrm{P}_{Q}(V=0)<1$.

Putting this together we see that, for $Q \in \mathcal{P}_{\text {lcc }}^{\circ}$, the rejection probability of the test $\phi_{+}$is strictly smaller than the preassigned level, and that it is strictly greater for $\phi_{-}$. This proves the upper part of (3.5) and the lower part of (3.7). A similar argument, with $\mathcal{P}_{\text {lcv }}^{\circ}$ instead of $\mathcal{P}_{\text {lcc }}^{\circ}$, provides the respective other parts.

Removing the qualifier 'strong' in the above arguments, which corresponds to the transition from $\mathcal{P}_{\text {lcv }}^{\circ}$ to $\mathcal{P}_{\text {lcv }}$ or from $\mathcal{P}_{\text {lcc }}^{\circ}$ to $\mathcal{P}_{\text {lcc }}$, we obtain inequalities instead of strict inequalities, and this leads to the remaining statements in (3.4) and (3.6).

The conditioning on $V$ means that the tests are invariant under scaling in the sense that the decision will not change if we multiply the arrival and departure data by a fixed positive constant.

The above procedures can easily be adapted to the two-sided case, i.e. for testing $Q=\operatorname{Exp}(\eta)$ for some $\eta>0$ against $Q \in \mathcal{P}_{\text {lcv }}^{\circ} \cup \mathcal{P}_{\text {lcc }}^{\circ}$.

\section{Asymptotics}

In the previous sections we had a fixed finite sample size $n$ and the arrival times were also regarded as fixed. We now investigate the asymptotic behaviour of the procedures under the assumption that arrivals occur at the points of a Poisson process with constant rate $\lambda$. This is, roughly, the $\mathrm{M} / \mathrm{G} / \infty$ queueing model mentioned in the introduction, but we 'close admittance' after the arrival of the $n$th customer. Formally, we have a sequence $X=\left(X_{i}\right)_{i \in \mathbb{N}}$ of arrival times, with $X_{1}=0$ and $X_{i+1}-X_{i}, i \in \mathbb{N}$, independent random variables, with distribution $\operatorname{Exp}(\lambda)$. Furthermore, $\tilde{Y}_{i}=X_{i}+Z_{i}$, where the $Z_{i}, i \in \mathbb{N}$, are independent, and all the $Z_{i} \mathrm{~s}$ have distribution $Q$. The sequences $X$ and $\left(Z_{i}\right)_{i \in \mathbb{N}}$ are also assumed to be independent. Let $Y=\left(Y_{n, i}\right)_{n \in \mathbb{N}, i=1, \ldots, n}$ be such that $Y_{n, 1}, \ldots, Y_{n, n}$ are the order statistics for $\tilde{Y}_{1}, \ldots, \tilde{Y}_{n}$ (we need a second index here as later arrivals may depart earlier than previous arrivals-see the cases of Mozart and Tchaikovsky in our running example). Again, let

$$
V_{i}:=i-1-\#\left\{1 \leq j \leq i: Y_{i, j} \leq X_{i}\right\}
$$

be the number of customers in the system at the time of the $i$ th arrival (it is easy to see that we do not need a second index for the $V$-variables - the right-hand side of (4.1) does not change if $Y_{i, j}$ is replaced by $Y_{n, j}$ with an arbitrary $n \geq i$ ). Finally, let $\Pi_{n}$ be the correct matching for the first $n$ arrivals and departures. Our first limit result is conditional on the sequence $X$ and the triangular array $Y$.

Theorem 4.1. Suppose that $Q=\operatorname{Exp}(\eta)$, and set $\rho=\lambda / \eta$, Then, with probability 1 , as $n \rightarrow \infty$,

$$
\begin{gathered}
\frac{1}{n} \mathrm{E}\left[d_{\tau}\left(\mathrm{id}, \Pi_{n}\right) \mid X, Y\right] \rightarrow \frac{\rho}{2}, \\
\frac{1}{n} \operatorname{var}\left[d_{\tau}\left(\mathrm{id}, \Pi_{n}\right) \mid X, Y\right] \rightarrow \frac{\rho(\rho+3)}{12}, \\
\mathcal{L}\left(\frac{d_{\tau}\left(\mathrm{id}, \Pi_{n}\right)-\mathrm{E}\left[d_{\tau}\left(\mathrm{id}, \Pi_{n}\right) \mid X, Y\right]}{\operatorname{var}\left[d_{\tau}\left(\mathrm{id}, \Pi_{n}\right) \mid X, Y\right]^{1 / 2}} \mid X, Y\right) \stackrel{\mathrm{D}}{\rightarrow} N(0,1) .
\end{gathered}
$$

Proof. We need the stochastic dynamics of the sequence $V=\left(V_{i}\right)_{i \in \mathbb{N}}$ as given in [18, Chapter 3.2]: $V$ is an irreducible and aperiodic Markov chain with stationary distribution 
$\pi=\operatorname{Po}(\rho)$, and the moments $\mathrm{E} V_{i}^{l}, l \in \mathbb{N}$, converge to the corresponding moments of $\operatorname{Po}(\rho)$, where we have written $\operatorname{Po}(\rho)$ for the Poisson distribution with mean $\rho$.

By the ergodic theorem for Markov chains (see Theorem 2 of [10, Section I.15]), we then have, with probability 1 ,

$$
\begin{gathered}
\lim _{n \rightarrow \infty} \frac{1}{n} \sum_{i=1}^{n} V_{i}=\sum_{j=0}^{\infty} j \pi_{j}=\rho, \\
\lim _{n \rightarrow \infty} \frac{1}{n} \sum_{i=1}^{n} V_{i}\left(V_{i}+2\right)=\sum_{j=0}^{\infty} j(j+2) \pi_{j}=\rho^{2}+3 \rho, \\
\lim _{n \rightarrow \infty} \frac{1}{n} \sum_{i=1}^{n} V_{i}^{4}=\sum_{j=0}^{\infty} j^{4} \pi_{j}<\infty .
\end{gathered}
$$

Using this together with (2.15), we obtain the first two statements of the theorem. For the distributional limit, we start with (2.14), which in the current notation becomes

$$
\mathcal{L}\left(d_{\tau}\left(\mathrm{id}, \Pi_{n}\right) \mid X, Y\right)=\underset{i=1}{\star} \operatorname{unif}\left(\left\{0, \ldots, V_{i}\right\}\right) .
$$

By the Lyapunov condition for the central limit theorem (see, e.g. [7, Section 27]), it is now enough to show that

$$
\lim _{n \rightarrow \infty} \frac{\sum_{i=1}^{n} V_{i}^{4}}{\left((1 / 12) \sum_{i=1}^{n} V_{i}\left(V_{i}+2\right)\right)^{2}}=0
$$

with probability 1 . From (4.3) we find that the numerator is $O(n)$ with probability 1 ; for the denominator, we use (4.2).

We now consider the asymptotic behaviour of the tests in Section 3 in the above model. Let $\alpha \in(0,1)$ be fixed. We write $\phi_{n,+}$ and $\phi_{n,-}$ for the level- $\alpha$ tests based on the first $n$ arrivals and departures. The following result shows that these tests are consistent 'inside' their respective hypothesis and alternative.

Theorem 4.2. (a) If $Q \in \mathcal{P}_{\text {lcc }}^{\circ}$ then

$$
\lim _{n \rightarrow \infty} \mathrm{E}_{Q} \phi_{n,+}(\Pi, V)=0 \text { and } \lim _{n \rightarrow \infty} \mathrm{E}_{Q} \phi_{n,-}(\Pi, V)=1
$$

(b) If $Q \in \mathcal{P}_{\mathrm{lcv}}^{\circ}$ then

$$
\lim _{n \rightarrow \infty} \mathrm{E}_{Q} \phi_{n,-}(\Pi, V)=0 \text { and } \lim _{n \rightarrow \infty} \mathrm{E}_{Q} \phi_{n,+}(\Pi, V)=1 .
$$

Proof. For general $Q$, the sequence $\left(V_{i}\right)_{i \in \mathbb{N}}$ will no longer be a Markov chain, but it still has a regenerative property in the sense that, with $v_{0}:=1$ and

$$
v_{k+1}:=\inf \left\{i>v_{k}: V_{i}=0\right\} \text { for all } k \in \mathbb{N},
$$

the blocks $\left(W_{k}\right)_{k \in \mathbb{N}}$, where

$$
W_{k}:=\left(V_{v_{k-1}}, V_{v_{k-1}+1}, \ldots, V_{v_{k}-1}\right),
$$

are independent and identically distributed. This is a consequence of the decomposition of $\mathrm{M} / \mathrm{G} / \infty$ queues into busy and idle periods; in particular,

$$
\mathrm{P}\left(v_{k}<\infty \text { for all } k \in \mathbb{N}\right)=1 .
$$


The blocks induce a decomposition of the set of permissible permutations. (In our running example we have chosen the composers to demonstrate this. Obviously, for $\pi$ to be permissible, we need $\pi(\{1,2,3,4,5\})=\{1,2,3,4,5\}$ and $\pi(\{6,7\})=\{6,7\}$.) Consequently, if $n=v_{k}-1$ for some $k \in \mathbb{N}$ then any $\pi \in \mathbb{S}_{n}^{\circ}$ can be written as

$$
\pi=\pi^{k-1} \circ \pi^{k-2} \circ \cdots \circ \pi^{1}
$$

where $\pi^{l}$ permutes the integers $v_{l-1}, v_{l-1}+1, \ldots, v_{l}-1$ only. This in turn leads to an additive decomposition of the $\tau$-distance,

$$
d_{\tau}(\mathrm{id}, \pi)=\sum_{l=1}^{k-1} d_{\tau}\left(\mathrm{id}, \pi^{l}\right) .
$$

Conditionally on $V$, we thus obtain a representation of the test statistics,

$$
d_{\tau}\left(\mathrm{id}, \Pi_{v_{k}-1}\right)=\sum_{l=1}^{k-1} \xi_{l}
$$

where the $\xi_{l}$ s are independent and identically distributed. By the law of large numbers we therefore have, conditionally on $V$,

$$
\lim _{k \rightarrow \infty} \frac{1}{v_{k}} d_{\tau}\left(\mathrm{id}, \Pi_{v_{k}}\right)=\mathrm{E}_{Q}\left[\xi_{1} \mid V\right]=: \chi(Q) \quad \text { with probability } 1 .
$$

The strict stochastic order of the distance distributions that we used in the proof of Theorem 3.1 implies that $\chi(Q)<\chi(\operatorname{Exp}(\eta))$ whenever $Q \in \mathcal{P}_{\text {lcc }}^{\circ}$. In connection with the conditional asymptotic normality in Theorem 4.1 this means that the conditional probability that $\phi_{v_{k},+}$ rejects will tend to 0 as $k \rightarrow \infty$, and that the conditional probability that $\phi_{\nu_{k},-}$ rejects will tend to 1 as $k \rightarrow \infty$, both with probability 1 .

In order to be able to uncondition, i.e. to integrate out the sequence $V$, we need to pass from the sequence $\left(v_{k}\right)_{k \in \mathbb{N}}$ of random times to the full deterministic sequence $1,2, \ldots$ This, however, is straightforward, using

$$
d_{\tau}\left(\mathrm{id}, \Pi_{v_{k}-1}\right) \leq d_{\tau}\left(\mathrm{id}, \Pi_{n}\right) \leq d_{\tau}\left(\mathrm{id}, \Pi_{v_{k+1}-1}\right)
$$

on $v_{k}-1 \leq n<v_{k+1}-1$, and the fact that $v_{k+1} / v_{k} \rightarrow 1$ with probability 1 as $k \rightarrow \infty$.

Taken together this proves (a). The arguments for the second part are similar.

Specialized to the parametric situations in (1.1) and (1.2), the theorem gives

$$
\lim _{n \rightarrow \infty} \mathrm{E}_{\beta, \eta} \phi_{+}(\Pi, V)= \begin{cases}0, & \beta>1, \\ \alpha, & \beta=1, \\ 1, & \beta<1,\end{cases}
$$

and a similar statement holds for $\phi_{-}$.

\section{Acknowledgements}

We wish to thank Ludwig Baringhaus for helpful discussions. We are also grateful to the anonymous referee for drawing our attention to [18].

This work has been supported by grants from the Deutsche Forschungsgemeinschaft and the State of Lower Saxony. 


\section{References}

[1] An, M. Y. (1998). Logconcavity versus logconvexity: a complete characterization. J. Econom. Theory 80, 350369.

[2] Asmussen, S. (2003). Applied Probability and Queues, 2nd edn. Springer, New York.

[3] Bagnoli, M. And Bergstrom, T. (2005). Log-concave probability and its applications. Econom. Theory 26, 445-469.

[4] Bai, Z. And Hsing, T. (2005). The broken sample problem. Prob. Theory Relat. Fields 131, 528-552.

[5] Barlow, R. E. and Proschan, F. (1975). Statistical Theory of Reliability and Life Testing. Holt, Rinehart and Winston, New York.

[6] BäUerle, N. AND GrüBel, R. (2005). Multivariate counting processes: copulas and beyond. ASTIN Bull. 35, 379-408.

[7] Billingsley, P. (1986). Probability and Measure, 2nd edn. John Wiley, New York.

[8] Bingham, N. H. And Pitts, S. M. (1999). Non-parametric estimation for the M/G/ $\infty$ queue. Ann. Inst. Statist. Math. 51, 71-97.

[9] Buchmann, B. And Grübel, R. (2003). Decompounding: an estimation problem for Poisson random sums. Ann. Statist. 31, 1054-1074.

[10] Chung, K. L. (1967). Markov Chains With Stationary Transition Probabilities. Springer, New York.

[11] DeGroot, M. H. and Goel, P. K. (1980). Estimation of the correlation coefficient from a broken random sample. Ann. Statist. 8, 264-278.

[12] DeGroot, M. H., Feder, P. L. And Goel, P. K. (1971). Matchmaking. Ann. Math. Statist. 42, $578-593$.

[13] Diaconis, P. (1988). Group Representations in Probability and Statistics (IMS Lecture Notes Monogr. Ser. 11). Institute of Mathematical Statistics, Hayward, CA.

[14] Hall, P. AND PARK, J. (2004). Nonparametric inference about service time distribution from indirect measurements. J. R. Statist. Soc. B 66, 861-875.

[15] Jungnickel, D. (1999). Graphs, Networks and Algorithms. Springer, Berlin.

[16] Lehmann, E. L. (1959). Testing Statistical Hypotheses. John Wiley, New York.

[17] Müller, A. And Stoyan, D. (2002). Comparison Methods for Stochastic Models and Risks. John Wiley, Chichester.

[18] TAKÁcs, L. (1962). Introduction to the Theory of Queues. Oxford University Press, New York. 